\title{
[DC] Self-Adaptive Technologies for Immersive Trainings
}

\author{
Joris Heyse* \\ Ghent University - imec, IDLab
}

\begin{abstract}
Online learning is the preferred option for professional training, e.g. Industry 4.0 or e-health, because it is more cost efficient than on-site organisation of realistic training sessions. However, current online learning technologies are limited in terms of personalisation, interactivity and immersiveness that are required by applications such as surgery and pilot training. Virtual Reality (VR) technologies have the potential to overcome these limitations. However, due to its early stage of research, VR requires significant improvements to fully unlock its potential. The focus of this $\mathrm{PhD}$ is to tackle research challenges to enable VR for online training in three dimensions: (1) dynamic adaptation of the training content for personalised trainings, by incorporating prior knowledge and context data into self-learning algorithms; (2) mapping of sensor data onto what happens in the VR environment, by focusing on motion prediction techniques that use past movements of the users, and (3) investigating immersive environments with intuitive interactions, by gaining a better understanding of human motion in order to improve interaction. The designed improvements will be characterised though a prototype VR training platform for multiple use cases. This work will not only advance the state of the art on VR training, but also on online e-learning applications in general.
\end{abstract}

Index Terms: Human-centered computing-Human computer interaction (HCI) - Interaction paradigms-Virtual reality; Applied computing - Life and medical sciences-Health informatics

\section{INTRODUCTION}

Online training is one of the most extended manners of teaching and learning when the face-to-face alternative is neither feasible, due to distance, nor possible, due to the requirements of the training [4]. Training applications in the industrial (Industry 4.0), medical (telesurgery), or educational (remote learning) domains already choose the online option. However, currently used online training systems present limitations in terms of personalisation, immersiveness and real-time assessment of the users/trainees. Virtual Reality (VR) is the key enabler for the next generation of online training systems [6]. VR will not only reduce the cost of the training, but also increase effectiveness and efficiency of the online training systems [1].

In contrast to a traditional online learning scenario, where the trainee interacts with the content only by means of a screen and a computer (attached to a content provider), in a VR-enabled training environment, as visualised in figure 1 , the trainee is immersed within the $360^{\circ}$ content by means of a Head Mounted Display (HMD). The HMD consists of hardware and software for precise head movement tracking, and two high resolution displays. The images on the displays show the content in the Field of View (FoV) of the user and are adapted based on the position of the head [5]. In addition, these VR systems can be extended by means of eye and body motion tracking sensors. The analysis of data from these sensors allows to improve immersion and training effectiveness, thus providing further

*e-mail: joris.heyse@ugent.be

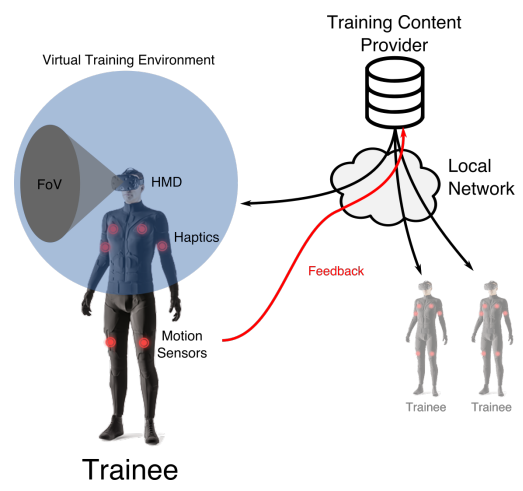

Figure 1: A vision of the ideal VR immersive training system that will be realised.

personalisation/adaptation for VR training systems [6]. Furthermore, VR-enabled training systems are envisioned to integrate kinesthetic feedback by means of haptics, further increasing the interaction with, and control of the training environment by the trainee. Due to the early stage of research for immersive technologies, the application of VR and haptics to online training is still far from unlocking its full potential. We identify three different areas of open research:

1. Personalisation of VR content: In order to achieve full immersiveness and effectiveness, the content needs to be personalised and adapted to the requirements of the trainee and the exercise.

2. Motion monitoring and tracking: The sensors installed on the HMD and body can provide valuable information for the adaptation of the training content and feedback for the trainer. However, nowadays, there is still no unambiguous mapping between sensor data and virtual environment.

3. Interactivity: The interaction with the environment and among users lacks realism, precision and real-timeliness it requires.

The purpose of this $\mathrm{PhD}$ research is to tackle the open challenges within the three above mentioned research areas. The remainder of this paper provides a thorough description of the defined goals of this research and the current status.

\section{Research goals}

In addition to the three defined research directions (personalisation, motion tracking and interactivity), there is a need for novel evaluation techniques able to create and evaluate these VR-enabled online training environments. Given the early stage of research and the challenge of integrating the technologies in training environments, we believe the development of a prototype VR training platform is a research line in itself. The design of a prototype VR training platform is will allow me to evaluate the research technologies on multiple use cases. Figure 2 presents how all four research components fit together. 


\subsection{VR Content Adaptation and Personalisation}

The first components focusses on personalisation of VR trainings. Current solutions require human trainers to decide the difficulty, the amount of guidance and the focus of the training exercise which is too expensive. This entails that a system will be developed that is able to select which exercise should be performed next by the user. Initially, self-learning algorithms such as Reinforcement Learning (RL) and data analytics are applied to get an understanding of how users relate to exercises. Another valuable source of information for personalising the VR training, are actual human trainers. However, because we want to create a system that does not require constant supervision of the trainer, the knowledge and expertise of these trainers has to be captured in a structured way so it can be used and applied later on, for many different cases. Some users also learn in very different ways, we hypothesise that clusters exist of trainees that learn in a similar way. An RL agent for each cluster would improve the adaptation performance for the users in each cluster especially in combination with the extracted expert knowledge.

\subsection{Motion Monitoring and Predicting}

The second research component is about recognition and prediction of the user motion. It is paramount to understand exactly how the user moves in reality in order to reflect that motion in virtual reality and react appropriately on it. Sensors available for VR systems are either, scares, expensive or to limited in use. Advanced techniques can use limited sensor data to approximate the actual movement of the user. This research investigates in several steps how to accurately predict and recognise motion from users, specifically to predict on what the attention of the user is focussed. First, by analysing HMD sensor data to predict in the near future where a user will be watching. Second, by incorporating additional sensor data from sensors all over the body for which hand motion will be especially interesting. This additional data can support better temporal prediction and more accurate spacial predictions.

\subsection{Interaction with the Environment and Among Users}

The third research challenge focusses on interaction, because interaction in VR is crucial for an immersive experience. However, intuitive interaction with both the environment and other users is a challenging topic. Interaction includes both the way in which an interaction is initiated with a person or object and the way in which this object responses to this action. The distinction is made between interaction of the user with its environment and interaction between multiple users within the same VR environments. Much like the training content adapts based on the goal of the training, the environment should adapts based on the intention of the user. This builds further on the results from the previous challenge which provides predictions of where a user is looking. The adaptive system, in turn makes changes to the environment or provide some sort of feedback. How the environment changes greatly depend on the use case of that environment. Haptics offer an appealing method for providing feedback on these interactions. However, matching the haptic signal with the interaction is non-trivial. For interaction between users, I focus on methods for distributing data between users. For example, the focus point of an instructor should shared with all users.

\section{Current Status}

The obtained results from this research will be applied on specific use cases of VR training. Currently, we have a project running in which a Virtual Reality Exposure Therapy (VRET) system is being researched and developed. This system will help people with severe anxieties to learn handle their fear. The so called, PATRONUS project 1 , aims to deliver a system that suggest automatic adaptation

\footnotetext{
${ }^{1}$ https://www.imec-int.com/en/what-we-offer/research-
} portfolio/patronus

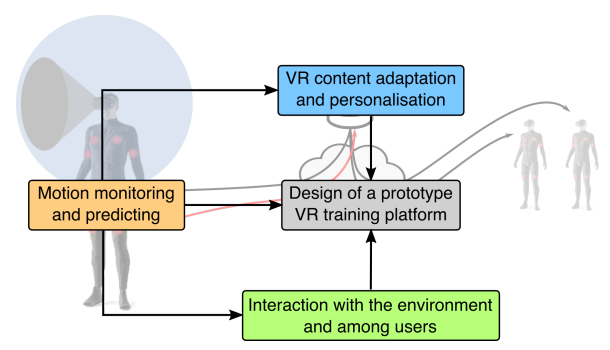

Figure 2: Overview of the tackled research challenges and their interconnection.

to the environment based on physiological data that is translated into an anxiety measurement. Within this project my focus is on the personalised and automated adaptation of the environment based on the profile of the user and the anxiety measure [2].

Secondly, we have developed a testbed for adaptive streaming of VR video [3] which will allow to further research the streaming techniques for the VRET use case. The core of the adaptive streaming technologie is accurate viewpoint prediction which also is the focus of the the second challenge. Currently, I am experimenting with reinforcement learning (RL) for predicting this user motion for which some preliminary results are available that have not been published yet.

\section{Conclusion}

VR is the best suited technology to enable a new generation of online training applications, in which the content is interactive, adaptive and personalised to the trainee's needs. However, bringing VR technologies to the required level by online VR training calls for improvement actions. The purpose of this $\mathrm{PhD}$ is to investigate, research, design, develop and evaluate tools, systems and algorithms to improve VR technologies in three identified areas: VR content personalisation, motion monitoring and tracking and interactivity. This work aims not only to advance the state of the art on VR training, but also on online e-learning applications in general.

Viewpoint prediction is currently a very hard problem, my early research on the topic revealed that users often behave unpredictably. Some new insights on the topic would help me further on track in improving the prediction performance. Additionally, natural interaction and behaviour of the environment in response to a user is a very wide and open challenge. This DC could help me narrow down this challenge in order to focus my research on a more specific area.

\section{REFERENCES}

[1] J. E. Brough, M. Schwartz, S. K. Gupta, D. K. Anand, R. Kavetsky, and R. Pettersen. Towards the development of a virtual environment-based training system for mechanical assembly operations. Virtual reality, 11(4):189-206, 2007.

[2] J. Heyse, F. Ongenae, F. D. Backere, and F. D. Turck. Personalisation of exercises in vret. In The Workshops of the Thirty-Second AAAI Conference on Artificial Intelligence, 2018.

[3] J. Heyse, M. T. Vega, T. Wauters, F. D. Backere, and F. D. Turck. Effects of Adaptive Streaming Optimizations on the Perception of 360 Virtual Reality Video. In 2018 30th International Teletraffic Congress (ITC 30), pp. 89-92, 2018.

[4] N. Jayakumar, O. Brunckhorst, P. Dasgupta, M. S. Khan, and K. Ahmed. e-learning in surgical education: a systematic review. JSE, 72(6):11451157, 2015.

[5] E. Peek, B. Wünsche, and C. Lutteroth. Virtual reality capabilities of graphics engines. 2013

[6] N. Vaughan, B. Gabrys, and V. N. Dubey. An overview of self-adaptive technologies within virtual reality training. Comput. Sci. Rev., 22:65-87, 2016. 